\title{
The rationale for testing drug combinations in aging in- tervention studies
}

\author{
Kavita Sharma ${ }^{a, \#}$, Juan Wang ${ }^{a, \#}$, Zhou Jiang ${ }^{a}$, Jenna Klug ${ }^{a}$, Martin Darvas ${ }^{b}$, Denise M. Imai ${ }^{c}$, Timothy \\ Snider ${ }^{\mathrm{d}}$, Laura Niedernhofer ${ }^{\mathrm{e}}$, Warren Ladiges ${ }^{\mathrm{a},{ }^{*}}$ \\ ${ }^{a}$ Department of Comparative Medicine, School of Medicine, University of Washington, Seattle, WA 98195, USA. \\ ${ }^{b}$ Department of Pathology, School of Medicine, University of Washington, Seattle, WA 98195, USA. \\ C Comparative Pathology Laboratory, University of California, Davis, CA, USA. \\ ${ }^{d}$ Department of Veterinary Pathobiology, Oklahoma State University, Stillwater, OK, USA. \\ ${ }^{e}$ Institute on the Biology of Aging and Metabolism and Department of Biochemistry Molecular Biology and Biophysics, \\ University of Minnesota, Minneapolis, MN 55455, USA.
}

\begin{abstract}
Aging is a complex process driven by seven intertwined pillars that functionally decline with increasing chronologic age. These pillars of aging include stem cell function, mitochondrial function, proteostasis, autophagy, nutrient sensing, metabolism, epigenetic control, and adaptation to stress, macromolecular damage and inflammation. All of the pillars appear to be interconnected such that a change in one, impinges upon others. With so many pillars of aging, it makes drug development to target aging processes equally complex. This leads to the notion that multiple pathways or biological processes need to be targeted to effectively prevent, delay or attenuate aging. The concept of drug combinations as a powerful anti-aging platform is intriguing but has yet to be tested systematically. Insulin function, mTOR (mammalian target of rapamycin) signaling and epigenetic regulation are well-established molecular pathways involved in the pathobiology of aging. Existing drugs that target these pathways include acarbose, rapamycin, and phenylbutyrate, respectively. Acarbose and rapamycin, used as single agents, extend the lifespan of mice. Thus, a cocktail of these drugs with different mechanisms of action would be expected to complement one another and robustly enhance a delay of aging and age-related disease not achievable with mono-therapeutic approaches. Studies to test this concept will be helpful in the development of clinical trials to enhance healthy aging.
\end{abstract}

Keywords: Aging intervention, drug combinations, glucose regulation, mTOR signaling, epigenetics

\section{Aging processes can be targeted with repur- posed drugs}

Aging is a complex multifactorial process involving multiple pathways that may need to be targeted to effectively prevent or slow aging [1]. A number of molecular pathways are well known for regulating lifespan, including insulin signaling, mTOR (mammalian target of rapamycin)

\footnotetext{
${ }^{\#}$ Contributed equally to this manuscript.

* Corresponding author: Warren Ladiges

Mailing address: Department of Comparative Medicine, School of Medicine, University of Washington, Seattle, WA 98195, USA. Email: wladiges@uw.edu
}

Received: 07 December 2019 / Accepted: 09 December 2019 signaling, and epigenetic regulation by histone deacetylation (HDAC). Selection of these specific pathways for a multiplex approach is based on their well-established roles in aging and age-related diseases, the fact that they can be targeted by existing, well-characterized and clinically efficacious drugs, and their distinct roles in aging and agerelated diseases leading to the potential for additive or synergistic effects when simultaneously therapeutically targeted (Figure 1).

Insulin signaling is influenced by a number of factors in an age-related manner. For example, age-related insulin resistance is due in part to increased adiposity and decreased efficiency of clearing misfolded proteins in the endoplasmic reticulum vital for proper function of insulin $[2,3]$. The mTOR pathway is sensitive to nutrient intake [4], and in an age-related manner enhances cell growth and proliferation [5], while suppressing vital autophagy and proteasome-mediated turn-over activities 


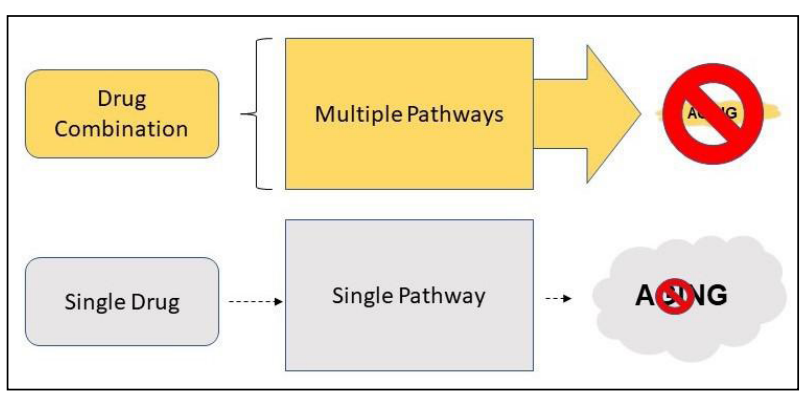

Figure 1. A drug combination that targets multiple pathways of aging has much more potential of suppressing aging than a single drug that only targets a single pathway.

[6]. Epigenetic dysregulation is implicated in a wide variety of age-related diseases [7]. The two major epigenetic mechanisms that influence gene expression during aging are DNA methylation and modification of histones that package DNA. Among the known histone modifications, acetylation has the greatest potential to induce chromatin unfolding and allow increased transcription. Deacetylases on the other hand, cause a more condensed, transcriptionally repressed chromatin conformation and become more active with increasing age [8]. Each of the three systems has been targeted with individual drugs, but only a few of these drugs have anti-aging affects, and no single drug has been shown to target all three pathways. Therefore, combinations of these drugs and the approach of simultaneously targeting multiple pillars of aging is anticipated to impact health span and lifespan greater than any single agent [9].

\section{Selection of drug combinations is based on physiological and molecular effects of each in- dividual drug}

The criteria for selecting which drugs to target these pathways as a combination approach in mouse studies must be based on well-validated preclinical evidence of anti-aging effects of individual drugs either by lifespan extension, for example by the NIA-supported Intervention Testing Program, or health span enhancement, or both. A relatively small number of drugs fit these criteria and three drugs can be identified to form the basis of a prototype cocktail: acarbose (Acb) [10], rapamycin (Rap) [11-13] and phenylbutyrate $(\mathrm{Pba})$ [14]. All three are clinically approved drugs with extensive biological, clinical, and safety data.

Acb has an antihyperglycemic effect resulting from competitive, reversible inhibition of membrane-bound intestinal alpha-glucosidase and pancreatic alpha-amylase, two enzymes needed to digest complex carbohydrates [15]. In diabetic patients, inhibiting these enzymes results in delayed glucose absorption and a lowering of postprandial hyperglycemia. Acb is considered a very safe and effective drug with only a fraction of patients experiencing side effects consisting of intestinal gas and diarrhea. Acb does not inhibit lactase and consequently does not induce lactose intolerance. Acb does not affect fasting blood glucose concentrations significantly or affect insulin secretion, so it does not cause hypoglycemia when given as monotherapy [16]. However, as a precaution, blood glucose and glycosylated hemoglobin HbA1c are usually monitored. It is contraindicated in diabetic ketoacidosis and inflammatory bowel disease. In the NIA Intervention Testing Program, acarbose increased medium and maximal lifespan in HET3 mice when treatment was started at 4 months of age [1] and at 16 months of age [17]. Tong et al [18] treated SAMP8 mice with Acb for six months and showed an alleviation of age-related behavioral and biochemical changes. Acb prevents cardiac ischemia/reperfusion injury in mice caused by post-prandial hyperglycemia [19].

Rap primarily targets complex 1 of TOR (TORC1), which is regulated by upstream pathways responsive to nutrient intake such as carbohydrates and amino acids [4]. High nutrients leading to TORC1 activation translates to rapid cell growth and proliferation, while a rapamycinmediated reduction in TORC1 signaling may phenocopy dietary restriction, which is known to enhance longevity in numerous model organisms. Downstream effects of reduced TORC1 signaling include enhanced autophagy and proteasome-mediated turnover [20, 21]. Rap and derivatives are currently being tested in a wide range of clinical trials for numerous chronic diseases. Long term rapamycin treatment results in several side effects including hyperglycemia and dyslipidemia, and testicular atrophy [12]. Transient short-term treatment may alleviate these side effects by delivering less drug for a shorter period of time [22]. A geropathology grading platform [23] was used to compare lesion scores in C57BL/6 male mice treated with Rap for two months, starting treatment at 24 months of age. Micro-encapsulated Rap was delivered in the feed at $42 \mathrm{ppm}$, and no difference in amount of food ingested in any of the cohorts was detected over the course of treatment. Mice treated with Rap had significantly lower lesion scores compared to control mice [24, 25].

$\mathrm{Pba}$ is a broad-spectrum inhibitor of class 1 and class 2 HDACS (both classes have zinc-driven catalytic sites) and inhibits histone deacetylation such that expression of genes associated with an overall slowing of cell growth and proliferation [7]. It is of interest that class 3 HDACS include the sirtuins, which have an absolute requirement for $\mathrm{NAD}+$, and are targeted by activators rather than inhibitors such as $\mathrm{Pba}$. $\mathrm{Pba}$ is also an active endoplasmic reticulum (ER) stress chaperone that enhances protein folding and decreases ER stress [26]. Therefore, there is rationale to consider $\mathrm{Pba}$ as part of a treatment strategy for heart disease, cancer, neurodegeneration, and inflammation. We have shown that $\mathrm{C} 57 \mathrm{BL} / 6$ mice treated with $\mathrm{Pba}$ for 12 months beginning at 4 months of age did not experience cognitive impairment associated with aging [26] and had decreased geropathology lesion scores in multiple organs (unpublished observations).

\section{A drug cocktail is a promising approach to en- hance healthy aging and delay age-related dis- eases by complementary interaction}


The rationale for combining the three drugs as a cocktail is based on expected mechanisms of molecular and cellular interaction [9]. Oral Acb will block intestinal alphaglucosidase so that carbohydrates are not broken down and absorbed. This will lower blood glucose levels and prevent postprandial insulin spikes. The lower blood glucose and decreased need for insulin will activate AMPK, which blocks mTORC1, the drug target for Rap. The lowered blood glucose provides less substrate for mitochondrial metabolism thereby stimulating mitochondria to increase electron transport chain (ETC) efficiency. The decreased demand for insulin helps alleviate insulin resistance induced by rapamycin-suppressed mTORC2. Rap will block mTORC1 signaling resulting in suppression of protein synthesis, suppression of mitochondrial metabolism, and activation of autophagy. The suppressed metabolic activity will increase ETC efficiency for ATP production and decrease the generation of ROS thought to drive macromolecular damage. The suppression of protein synthesis will conserve valuable cellular resources. Pba will activate genes involved in an overall slowing of cell growth and proliferation and will improve proper insulin maturation and more efficient insulin signaling. Activation of autophagy will help eliminate damaged macromolecules and organelles. The decreased substrate triggered by Acb and the downregulation of the mitochondrial proteasome by Rap will provide a cellular environment reminiscent of a more youthful phenotype.

In conclusion, drug combinations such as rapamycin, acarbose, and phenylbutyrate are a novel approach designed to complement mechanisms of action of each individual molecular target and robustly enhance a delay of aging and age-related disease not seen with mono-therapeutic approaches. This type of drug cocktail could serve as a powerful prototype for developing future aging intervention strategies, but rigorous preclinical and clinical studies are still needed to assure efficacy and safety especially in older populations.

\section{Declarations}

Conflict of Interest: Warren Ladiges is a member of the Editorial Board of Aging Pathobiology and Therapeutics. All authors declare no conflict of interest and were not involved in the journal's review or desicions related to this manuscript.

\section{References}

1. Ladiges W. The quality control theory of aging. 2014.

2. Ladiges W, Morton J, Blakely C, et al. Tissue specific expression of PKR protein kinase in aging B6D2F1 mice. Mechanisms of ageing and development, 2000, 114(2): 123-132.

3. Ladiges W C, Knoblaugh S E, Morton J F, et al. Pancreatic $\beta$-cell failure and diabetes in mice with a deletion muta- tion of the endoplasmic reticulum molecular chaperone gene P58IPK. Diabetes, 2005, 54(4): 1074-1081.

4. L oewith R, Hall M N. Target of rapamycin (TOR) in nutrient signaling and growth control. Genetics, 2011, 189(4): 1177-1201.

5. Vellai T, Takacs-Vellai K, Zhang Y, et al. Genetics: influence of TOR kinase on lifespan in C. elegans. Nature, 2003, 426(6967): 620.

6. Kapahi P, Zid B M, Harper T, et al. Regulation of lifespan in Drosophila by modulation of genes in the TOR signaling pathway. Current Biology, 2004, 14(10): 885-890.

7. Pasyukova E G, Vaiserman A M. HDAC inhibitors: A new promising drug class in anti-aging research. Mechanisms of ageing and development, 2017, 166: 6-15.

8. Walsh M E, Van Remmen H. Emerging roles for histone deacetylases in age-related muscle atrophy. Nutrition and healthy aging, 2016, 4(1): 17-30.

9. Ladiges W, Liggitt D. Testing drug combinations to slow aging. 2018.

10. Harrison D E, Strong R, Sharp Z D, et al. Rapamycin fed late in life extends lifespan in genetically heterogeneous mice. nature, 2009, 460(7253): 392.

11. Harrison D E, Strong R, Allison D B, et al. Acarbose, 17- $\alpha$ estradiol, and nordihydroguaiaretic acid extend mouse lifespan preferentially in males. Aging cell, 2014, 13(2): 273-282.

12. Wilkinson J E, Burmeister L, Brooks S V, et al. Rapamycin slows aging in mice. Aging cell, 2012, 11(4): 675-682.

13. Flynn J M, O'Leary M N, Zambataro C A, et al. Late-life rapamycin treatment reverses age-related heart dysfunction. Aging cell, 2013, 12(5): 851-862.

14. Walsh M E, Bhattacharya A, Sataranatarajan K, et al. The histone deacetylase inhibitor butyrate improves metabolism and reduces muscle atrophy during aging. Aging Cell, 2015, 14(6): 957-970.

15. Balfour J A, McTavish D. Acarbose. An update of its pharmacology and therapeutic use in diabetes mellitus. Drugs, 1993, 46(6): 1025-1054.

16. Yamamoto M, Otsuki M. Effect of inhibition of alphaglucosidase on age-related glucose intolerance and pancreatic atrophy in rats. Metabolism, 2006, 55(4): 533540.

17. Strong R, Miller R A, Antebi A, et al. Longer lifespan in male mice treated with a weakly estrogenic agonist, an antioxidant, an $\alpha$-glucosidase inhibitor or a Nrf2-inducer. Aging cell, 2016, 15(5): 872-884.

18. Tong J J, Chen G H, Wang F, et al. Chronic acarbose treatment alleviates age-related behavioral and biochemical changes in SAMP8 mice. Behavioural brain research, 2015, 284: 138-152.

19. Frantz S, Calvillo L, Tillmanns J, et al. Repetitive postprandial hyperglycemia increases cardiac ischemia/reperfusion injury: prevention by the $\alpha$-glucosidase inhibitor acarbose. The FASEB journal, 2005, 19(6): 591-593.

20. Powers R W, Kaeberlein M, Caldwell S D, et al. Extension of chronological life span in yeast by decreased TOR pathway signaling. Genes \& development, 2006, 20(2): 174-184. 
21. Johnson S C, Rabinovitch P S, Kaeberlein M. mTOR is a key modulator of ageing and age-related disease. Nature, 2013, 493(7432): 338-345.

22. Arriola Apelo S I, Neuman J C, Baar E L, et al. Alternative rapamycin treatment regimens mitigate the impact of rapamycin on glucose homeostasis and the immune system. Aging cell, 2016, 15(1): 28-38.

23. Snyder J M, Snider T A, Ciol M A, et al. Validation of a geropathology grading system for aging mouse studies. GeroScience, 2019, 41(4): 455-465.

24. Ladiges W, Snyder J M, Wilkinson E, et al. A new preclini- cal paradigm for testing anti-aging therapeutics. Journals of Gerontology Series A: Biomedical Sciences and Medical Sciences, 2017, 72(6): 760-762.

25. Bell-Temin H, Yousefzadeh M J, Bondarenko A, et al. Measuring biological age in mice using differential mass spectrometry. Aging (Albany NY), 2019, 11(3): 1045.

26. Wiley J C, Pettan-Brewer C, Ladiges W C. Phenylbutyric acid reduces amyloid plaques and rescues cognitive behavior in AD transgenic mice. Aging Cell, 2011, 10(3): 418-428.

Cite this article as: Sharma $\mathrm{K} \mathrm{C}$, Wang J, Jiang Z, et al. The rationale for testing drug combinations in aging intervention studies[J]. Aging Pathobiology and Therapeutics, 2019, 1(1): 01-04. 